\title{
Formación de la ciudad global, economía inmobiliaria y transnacionalización de espacios urbanos. El caso de Ciudad de México
}

\section{Christof Parnreiter}

Instituto de Geografía, Universidad de Hamburgo.

RESUMEN | Desde los años 1990, el mercado de oficinas en la Ciudad de México se ha transformado fundamentalmente. La superficie de oficinas se duplicó a 6.9 mill. $\mathrm{m}^{2}$ desde 1997 , con el $80 \%$ de la actividad constructora recayendo en el segmento de mercado de alta calidad. Además, la geografía del mercado cambió, ya que se crearon nuevos polos de desarrollo inmobiliario al poniente del Central Business District tradicional. El texto sostiene que la transformación de mercados inmobiliarios urbanos es motivado esencialmente por la "fuerza motriz" de procesos de formación de la ciudad global, dado que cada vez más empresas globales que tienen su sede (regional) en la Ciudad de México demandan más espacio de oficinas de alta calidad. En la segunda parte del texto se sostiene que las transformaciones del mercado de oficinas y de los espacios urbanos en la Ciudad de México se pueden comprender como "transnacionalización". Tomando la Torre Mayor como un estudio de caso, se demuestra que los nuevos espacios de la ciudad global están producidos y utilizados mayoritariamente por actores sin una identidad nacional claramente demarcada. Además, están construidos según nuevos mecanismos de planificación (Planeación Estratégica) que corresponden a un nuevo estándar global, por lo cual se sostiene que las reglas esenciales de la gestión urbana también están cada vez más desnacionalizadas.

PAlABRAS Clave | Ciudad global, mercado inmobiliario, planificación urbana, geografía económica

ABSTRACT | Since the 1990s, the office market in Mexico City has been fundamentally transformed. The available office space doubled since 1997 to 6.9 mill.m ${ }^{2}$, with the first class segment capturing $80 \%$ of the construction activity. In addition, the geography of the office market has been altered, because new centers of property development have been created in the Western parts of the city. The paper goes on to argue that the transformation of the office market is basically driven by processes of global city formation, because ever more global corporations have a (regional) headquarter in Mexico City, what has caused a sharp increase in the demand for first class office spaces. In the second part of the paper it is maintained that the transformation of the office market and of urban spaces in Mexico City are best understood as "transnationalization". Taking the Torre Mayor as a case study it is shown that the new global city spaces are produced and used principally by actors without a clear national identity. In addition, they have been built according to new mechanisms of planning (Strategic Planning), which correspond to a new global standard. Accordingly, it is sustained that the rules of urban planning and management are becoming denationalized, too.

KEY WORDS | Urban environment, environment quality, urban sustainability.

Recibido el 15 de noviembre de 2010, aprobado el 14 de marzo de 2011.

Correspondencia: Bundesstraße 55 (Geomatikum), D-20146 Hamburg. Tel.:+49 (0) 4042838-4961. E-mail: parnreiter@geowiss.uni-hamburg.de 


\section{La economía inmobiliaria como asunto de la investigación de la ciudad global}

Hasta el momento, la economía inmobiliaria es un asunto poco atendido en la investigación de la ciudad global ${ }^{1}$. Eso sorprende, considerando que Saskia Sassen ya hace 20 años advirtió en su libro "The Global City" (Sassen, 1991, 2001), que la formación de la ciudad global está relacionada con modificaciones en la economía inmobiliaria. Primero, el surgimiento de funciones de la ciudad global aumenta la demanda por oficinas de alta calidad, porque las empresas dedicadas a los servicios al productor requieren de una infraestructura condigna para poder captar deberes de control y administración en la economía global.

La consecuencia de este aumento de demanda es un boom de construcción, enfocado a ciertos barrios - por lo general en los centros- y ciertos sectores del mercado -los inmuebles oficina de primera clase. Por ende, desde los años 1980 "la rápida construcción de un complejo de oficinas de gran altura tras otro" (Sassen, 2001, p. 334) caracteriza a la transformación física de ciudades globales. Además, se está formando un mercado inmobiliario transfronterizo cuyas zonas centrales Sassen localiza en las ciudades globales. El aumento de la demanda por espacios de oficina de primera clase eleva los precios (principalmente en las localidades centrales), los desacopla del desarrollo económico de la ciudad y eso, a su vez, atrae tanto a inversores nacionales como extranjeros (Sassen, 2001, p. 190). Estudios demuestran que ante todo, aquellos centros urbanos que presentan un desarrollo dinámico en el área de servicios al productor, atraen a inversiones inmobiliarias transfronterizas (Fainstein, 2001). Esta tendencia de buscar inversiones rentables en mercados inmobiliarios urbanos se acelera en periodos de sobreacumulación, en los cuales las inversiones inmobiliarias asumen el papel de "una especie de última esperanza para encontrar usos productivos para la rápida acumulación de capital".

(Harvey, 1985, p. 20). Finalmente, el boom de inversiones inmobiliarias transfronterizas se intensificó aún más debido a que muchas empresas globales de servicios al productor (sobre todo aquéllas del sector financiero), al mismo tiempo, son propietarios y usuarios de las oficinas (Lizieri, Baum y Scott, 2000). Así, dichas empresas se convirtieron en actores determinantes en los segmentos de primera clase de los mercados de oficinas en numerosas ciudades globales.

Sin embargo, la relación entre la formación de la ciudad global y la internacionalización de un mercado inmobiliario local realmente no se ha investigado sistemáticamente, salvo para los casos de Nueva York y Londres (p.e. Fainstein, 2001). $\mathrm{Si}$ bien es cierto que otros estudios tienen ciertas ciudades globales (sobre todo las asiáticas) como objetos (Haila, 2000; Han, 2005; Yusuf y Wu, 2002), su referencia a la investigación de la ciudad global por lo general se limita a la absorción del término. Para América Latina, el fenómeno de la internacionalización de merca-

1 Mi definición de la ciudad global se basa en la conceptualización presentada por Sassen (Sassen 1991, 2001). Según esta autora, ciudades globales son centros de producción y sumistro de servicios al productor (como servicios financieros, legales, inmobiliarias, de contabilidad, asesoramiento de empresas, etc.), que son esenciales para el funcionamiento y la gestión de la economía global. Por eso, ciudades globales son centros de donde se organizan y controlan los multiples flujos transfronterizos que characterizan a la globalización. 
dos inmobiliarios se ha analizado desde varias perspectivas (ver, Sabatini y Cáceres, 2005; Libertun, 2006; Bernardes da Silva y Castillo, 2007; Valenzuela, 2007; Fuchs y Scharmanski, 2009). Sin embargo, en general estos estudios pasan sin referencia a procesos de la formación de la ciudad global. La postergación de la economía inmobiliaria por la investigación de la ciudad global sorprende porque los "city builders" (Fainstein, 2001) conceptualmente constituyen un eslabón entre los procesos de la globalización y las transformaciones espaciales que muchas ciudades vivían en las últimas décadas.

En el documento presente se analiza la "huella dactilar" que dejó la economía inmobiliaria en la Ciudad de México, y su relación con la formación de la ciudad global. La hipótesis es que la transformación en ciudad global causa la globalización de ciertos segmentos del mercado inmobiliario y, junto con ella, cambios marcados en la configuración física de la ciudad. Para examinar esta hipótesis, la Ciudad de México es un ejemplo aparejado, porque ambos fenómenos son relativamente recientes, por cuya causa se puede esculpir articuladamente si -y hasta qué grado- están relacionados con procesos de la formación de la ciudad global. A ese fin se relaciona el boom de construcción desde los años 1990, que ha resultado en la formación de una nueva geografía corporativa (Grant y Nijman, 2002), con la afluencia de empresas multinacionales, que tiene su origen en la formación de la ciudad global ${ }^{2}$. A continuación de este análisis se va a argumentar, tomando la Torre Mayor como ejemplo, qué partes de la Ciudad de México están viviendo un proceso de transnacionalización, lo que significa una desintegración parcial de su entorno local y nacional.

\section{La formación de la ciudad global en México}

La formación de una ciudad global implica que la ciudad atrae a actividades económicas que son necesarias para el funcionamiento de mercados globales y que la importancia de las mismas aumenta dentro de la economía de la ciudad. Ya que estas funciones de control y gestión sobre todo se ejercen por medio de servicios al productor, el crecimiento, la concentración y la articulación global de estos servicios se consideran como indicadores importantes de la formación de la ciudad global (Sassen, 2001).

Para la Ciudad de México está bien documentado que su papel económico ha cambiado paralelamente con la globalización de la economía en México -el epicentro de la economía nacional se convirtió en una "charnela" entre la producción nacional y el mercado mundial (Parnreiter 2002, 2010). Eso se ve, por ejemplo, en una concentración muy alta de los servicios al productor de la Ciudad de México, de donde viene el $76 \%$ del valor agregado nacional (cálculos propios a base de INEGI, 2004). Para el propósito de este texto es aún más explicativo que los servicios al productor están altamente centralizados no sólo en Ciudad de México, sino

Como geografía corporativa se entiende, según Grant y Nijman (2002), el patrón geográfico de la ubicación de las empresas grandes en una ciudad. 
también adentro de la ciudad. El 55\% del valor agregado mexicano se realiza en sólo tres delegaciones del Distrito Federal, es decir, en Cuauhtémoc, Miguel Hidalgo y en Benito Juárez (Cuadro 1).

CUADRO 1 | Servicios al productor, valor agregado, 2003, porcentaje de la producción nacional

\begin{tabular}{|c|c|c|c|c|c|}
\hline & $\begin{array}{l}\text { CD. DE } \\
\text { MÉxICo }\end{array}$ & $\begin{array}{c}\text { Distrito } \\
\text { FEDERAL }\end{array}$ & $\begin{array}{l}\text { BENITO } \\
\text { JUÁREZ }\end{array}$ & Cuauhtémoc & $\begin{array}{c}\text { Miguel } \\
\text { Hidalgo }\end{array}$ \\
\hline $\begin{array}{l}\text { TODOS LOS SERVICIOS } \\
\text { AL PRODUCTOR }\end{array}$ & 76.4 & 75.0 & 8.5 & 29.6 & 17.5 \\
\hline $\begin{array}{l}\text { SERVICIOS } \\
\text { FINANCIEROS Y DE } \\
\text { SEGUROS }\end{array}$ & 87.7 & 87.2 & 11.2 & 44.0 & 8.9 \\
\hline $\begin{array}{l}\text { SERVICIOS } \\
\text { INMOBILIARIOS }\end{array}$ & 32.5 & 26.4 & 3.1 & 3.7 & 7.3 \\
\hline $\begin{array}{l}\text { SERVICIOS } \\
\text { PROFESIONALES, } \\
\text { CIENTÍFICOS Y } \\
\text { TÉCNICOS }\end{array}$ & 50.1 & 46.4 & 7.3 & 8.0 & 18.5 \\
\hline $\begin{array}{l}\text { DIRECCIÓN DE } \\
\text { CORPORATIVOS Y } \\
\text { EMPRESAS }\end{array}$ & 80.6 & 79.7 & 0.3 & 0.3 & 66.2 \\
\hline
\end{tabular}

FUENTE CÁLCULOS PROPIOS A BASE DE INEGI, 2004

Cabe destacar que también la concentración de las casas matrices de las 500 empresas más grandes registradas en México está muy alta y, aún más importante, creciendo (expansión por varios años). El número de empresas grandes, que tienen sus sedes principales en la Ciudad de México, aumentó de manera continua y paralelamente al aumento y la concentración de servicios al productor. En 1993, un año antes de la entrada en vigor del Tratado de Libre Comercio (TLC), 256 de las 500 empresas más grandes tenían sus sedes principales en la Ciudad de México. Trece años más tarde ya fueron 352 - un aumento de 37\%. La tendencia de centralización también se muestra adentro de la ciudad: de las empresas top-500, que tenían sus sedes en la Ciudad de México en 1993, 85\% se estableció en el Distrito Federal -en el año 2003 ya eran 93\%. Dentro del Distrito Federal se anota una dislocación hacia el poniente. Mientras la delegación central de Cuauhtémoc con su Centro Histórico y una parte del "Paseo de la Reforma", y delegaciones del norte y nororientales que primordialmente están marcados por industrias, muy claramente van perdiendo importancia, Miguel Hidalgo, y en particular Álvaro Obregón y Cuajimalpa en el poniente de la ciudad van ganando numerosas sedes de empresas (Cuadro 2). En total, la focalización en la Ciudad de México aumenta con la grandeza de la empresa (volumen de ventas) y con el entrelazamiento global (exportaciones, 
el grado de participación de capital extranjero). Pues, de las empresas que (en su mayoría) son propiedad mexicana, un 63\% tiene su sede principal en la Ciudad de México, mientras que las empresas extranjeras tienen el $73 \%$.

CUADRO 2 | Localización de las casas matrices de las 500 empresas más grandes de México, 1993, 2006.

\begin{tabular}{|c|c|c|c|c|c|c|c|}
\hline & $\begin{array}{l}\text { CD. DE } \\
\text { MÉXICO }\end{array}$ & $\begin{array}{l}\text { DISTRITo } \\
\text { FEDERAL }\end{array}$ & $\begin{array}{l}\text { BENITO } \\
\text { JuÁREZ }\end{array}$ & Cuauhtémoc & $\begin{array}{c}\text { Miguel } \\
\text { Hidalgo }\end{array}$ & $\begin{array}{c}\text { ÁlVARo } \\
\text { OBREgón }\end{array}$ & Cuajimalpa \\
\hline 1993 & 256 & 217 & 17 & 49 & 75 & 20 & 5 \\
\hline 2006 & 352 & 329 & 27 & 47 & 113 & 56 & 24 \\
\hline 1993 & $100,0 \%$ & $85,0 \%$ & $6,7 \%$ & $19,2 \%$ & $29,4 \%$ & $7,8 \%$ & $1,9 \%$ \\
\hline 2006 & $100,0 \%$ & $93,4 \%$ & $7,6 \%$ & $13,3 \%$ & $32,1 \%$ & $15,9 \%$ & $6,8 \%$ \\
\hline
\end{tabular}

FUENTE CÁlCULOS PROPIOS A BASE DE EXPANSIÓN 1994, 2007.

\section{El mercado de oficinas}

El mercado de oficinas se expandió abultadamente en línea con la formación de la ciudad global (para lo siguiente, los datos provienen de los reportes más recientes de CB Richard Ellis, Colliers International, Cushman \& Wakefield y Jones Lang LaSalle). Entre 1997 y 2009, la superficie entera de oficinas se duplicó a 6.9 mill.m², cuando el $80 \%$ de la actividad constructora recae en el segmento de mercado de alta calidad. La clase A+ se expandió por más de $800,00 \mathrm{~m}^{2}$ entre 2001 y 2008, sumándose en 2008 al 30\% de la superficie entera de oficinas en la Ciudad de México (mientras en 2001 su participación ha sido el 19\%). Las clases A y A+ suman a más de 3 mill.m², lo que equivale a un $55 \%$ de todo el espacio de oficinas. Eso significa que la Ciudad de México tiene más espacios de oficinas de primera clase que las demás ciudades en América Latina (São Paulo tiene alrededor 2 mill.m², Rio y Santiago 1.5 mill.m² ${ }^{2}$.

El gran crecimiento de la oferta de inmuebles de oficina de alta calidad va acompañado por un cambio de la estructura espacial del mercado. En el poniente de la ciudad, y hasta cierto grado también en el sur, se formó un nuevo Central Business District (CBD), como ya se pudo suponer por la dislocación de las casas matrices de las empresas más grandes en México. En el año 2001, el CBD tradicional -el Centro Histórico, el Paseo de la Reforma aproximadamente hasta el anillo Periférico y a su norte Polanco así como Insurgentes y áreas circunvecinas- cubrió un 57\% de todos los espacios de oficina ${ }^{3}$. En 2008, la participación del CBD tradicional en todos los espacios de oficina ha bajado al $48 \%$, principalmente por una disminución

3 En la Cd. de México, empresas inmobiliarias operan con nueve submercados, cuya delimitación no se orienta en bordes de administración: Mientras Polanco y Lomas Palmas se encuentran en la delegación Miguel Hidalgo, otros mercados inmobiliarios se extienden a varias delegaciones: El corredor Centro-Reforma llega de Cuauthémoc hasta Miguel Hidalgo- y su alargamiento (Bosques de Lomas) llega de Miguel Hidalgo hasta Cuajimalpa. Santa Fe está situado en Àlvaro Obregón y Cuajimapla. El mercado Insurgentes aún cubre parte de cuatro delegaciones: Benito Juárez, Cuauthémoc, Àlvaro Obregón y Miguel Hidalgo, mientras Periférico Sur esta situado en Tlalpan y Magdalena Contreras. 
drástica de Polanco (Figura 1). Por el otro, tanto Santa Fe, cuya participación en el mercado de oficinas se expandió del 12 al 17\%, como Lomas Palmas (12\% en 2008) y Bosques de las Lomas (10\%) anotaron ganancias llamativas. Se ha, por ende, construido un nuevo CBD en el poniente de la ciudad.

FIGURA 1 | Localización de las empresas más grandes de México y mercado de oficinas en la Ciudad México

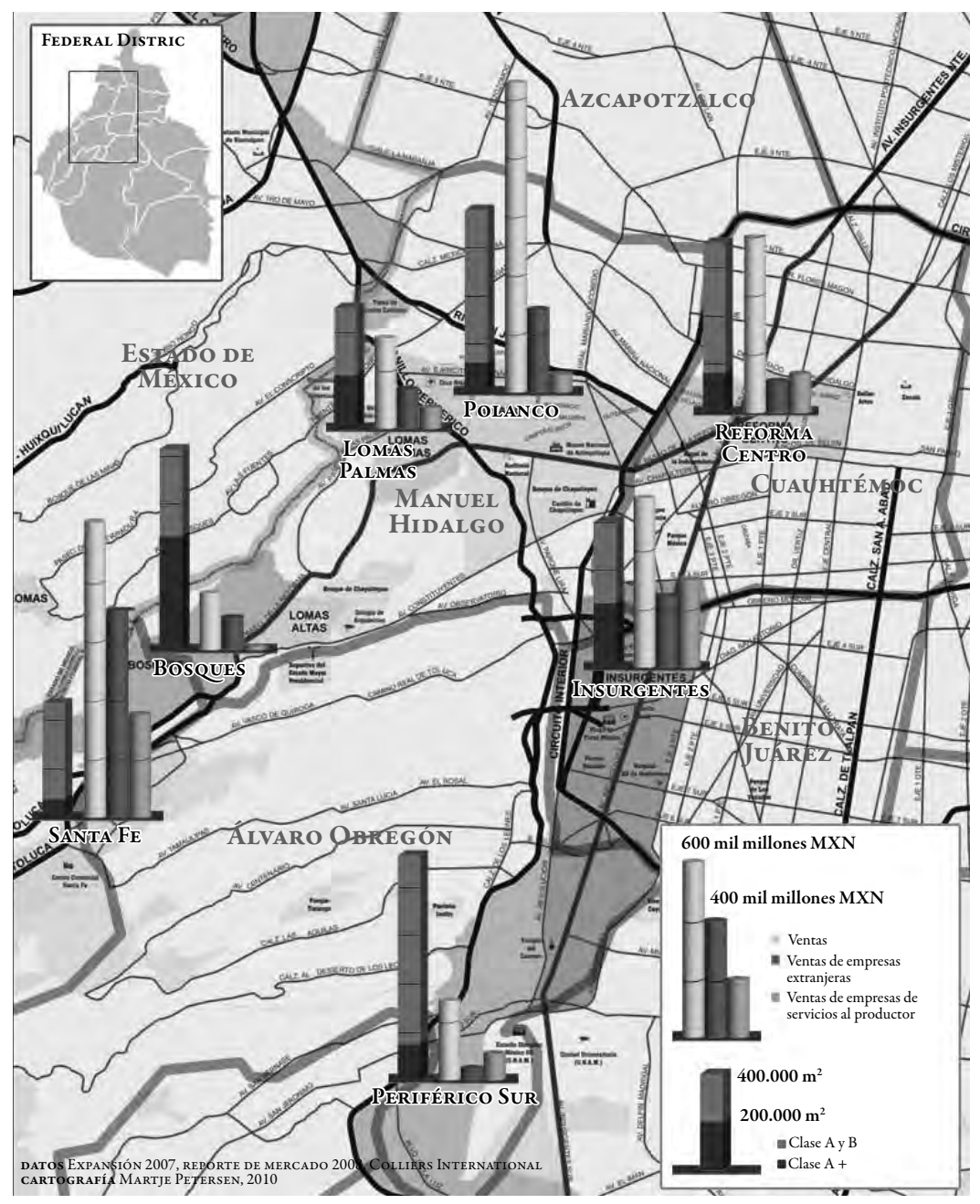

Los cambios en la estructura espacial del mercado de inmuebles de oficina se ven aún más claros si se enfoca solamente al segmento de alta calidad. En cuanto al CBD tradicional, Polanco e Insurgentes suman un porcentaje de espacio de ofici- 
nas A+ cada vez menor (la participación de Polanco se redujo del 15 al 8\%, la de Insurgentes del 19 al 10\%). El corredor Centro Histórico-Reforma, no obstante, está rápidamente aumentando su participación en el mercado de primera clase, casi triplicando su parte a $11 \%$. Sin embargo, la mayoría de las oficinas de alta calidad se ha construido en el poniente de la ciudad: Santa Fe cuenta casi con un tercio del inventario A+ (2001: 22\%; 2008: 32\%), Lomas Palmas con 18\%, y Bosques de las Lomas con $15 \%$. En estas zonas, las áreas A+ constituyen el segmento más importante del mercado (en Santa Fe componen el 56\% de todo el inventario, en Lomas Palmas y Bosques de las Lomas, 44\%). En el corredor Centro Histórico -en Paseo de la Reforma-, al contrario, pese al reciente boom de construcciones, sólo el $23 \%$ del espacio de oficinas pertenece a la clase $\mathrm{A}+$.

\section{Formación de ciudad global y la nueva geografía corporativa}

El análisis de las dinámicas del mercado de oficinas y de la ubicación de las empresas más grandes de México revela que no existe una relación directa entre la calidad de los inmuebles y las ventas de las empresas. De las diez empresas más grandes de México, ocho tienen su casa matriz en la Ciudad de México. Cinco de ellas (América Móvil, Comisión Federal de Electricidad, Teléfonos de México, General Motors, Telcel) se encuentran en Polanco y en el corredor Centro-Reforma, que componen sólo el 19\% del área A+ del mercado de oficinas. Santa Fe, al contrario, solamente hospeda a una empresa top-10 (Chrysler), mientras PEMEX y Wal-Mart, la empresa más grande de México y la cuarta, están ubicadas afuera de los mercados inmobiliarios más dinámicos. La imagen cambia, sin embargo, si se considera no solamente a las top-10 empresas. De las empresas top-50, Santa Fe hospeda a 11, mientras Polanco y el corredor Centro-Reforma llegan a 5 sedes de empresas cada uno.

Considerando la distribución espacial de las empresas según sus ventas se puede reconocer claramente, que la Ciudad de México entretanto tiene dos CBDs: 38\% de las ventas de las empresas top-500 con sede en México se contabilizan en el CBD tradicional, mientras el 31\% tiene su origen en el CBD nuevo (ver Figura 1). Cabe señalar que esta bifurcación tiene una dimensión temporal: mientras varias empresas grandes, que en los tiempos de sustitución de importaciones ya han jugado un papel muy importante en la economía mexicana (como por ejemplo, las empresas estatales o la mayoría de los consorcios de automóviles) están establecidas en el CBD tradicional, muchas empresas que se han asentado desde los años noventa favorecen el nuevo CBD con sus oficinas de mejor calidad.

Eso se ve claramente en la relación entre la distribución espacial de la superficie de oficinas de primera calidad, por un lado, y de las sedes de empresas de posesión extranjera y la ubicación de servicios al productor, por otro. Santa Fe tiene el número más grande de empresas extranjeras top-500 (28), seguido por Lomas Palmas (23) y Polanco (18). Al mismo tiempo, en Santa Fe se gana un tercio de las ventas de empresas extranjeras top-500, mientras en Polanco son el 13\% y de Lomas Palmas el $6 \%$. En cuanto a las sedes de empresas de servicios al productor, si bien es 
cierto que Santa Fe no tiene el número más alto (el corredor Centro-Reforma cuenta con 30, Lomas Palmas y Insurgentes con 24 cada uno, y Santa Fe sólo con 16) es importante destacar que en cuanto a las ventas Santa Fe está dominando muy claramente: allí se originan el $36 \%$ de las ventas de empresas de servicios al productor - contrastado con el 27\% en Insurgentes y el 12\% en el corredor Reforma-Centro. En total, el nuevo CBD cuenta con el $47 \%$ de las ventas de las empresas extranjeras y el $52 \%$ de las empresas de servicios al productor (ver Figura 1).

Por ende, la nueva geografía corporativa está marcada por un patrón particular de las empresas que en su mayor parte son de propiedad extranjera, y de las empresas de los servicios al productor. Ambas tienen otras preferencias de ubicación que las empresas mexicanas: Se instalan con mayor frecuencia en la Ciudad de México, y adentro de la ciudad favorecen a oficinas en Santa Fe y en otras partes del nuevo CBD. Eso sugiere que los procesos de formación de la ciudad global han modificado la demanda para oficinas en la Ciudad de México cuantitativa y cualitativamente. Una parte creciente de la demanda está creada por las empresas multinacionales como también las de los servicios al productor, que al mismo tiempo demandan, más que todo, oficinas de alta calidad.

La observación de que la demanda de oficinas de primera clase por parte de empresas multinacionales es un factor fundamental para el desarrollo del mercado inmobiliario y la construcción de un nuevo $\mathrm{CBD}$, se comprueba también por una tasa de disponibilidad en disminución desde el año 2002, incluso a pesar de un aumento de la actividad constructora. A finales de 2008, en plena crisis, la tasa de disponibilidad para oficinas de las clases A y A+ se mantuvo al $5 \%$, pero ha crecido desde entonces a una tasa entre 7 y $9 \%$. Esta tasa se encuentra, sin embargo, por debajo de las de muchas otras ciudades. Otra consecuencia del aumento de la demanda es el encarecimiento de las rentas en la última década. Aunque en 2007 los precios se mantuvieron en un nivel bajo comparado con el año 1997, muestran un movimiento expansivo continuo a partir del año 2002, que no fue interrumpido por la crisis $2008 / 9$. Finalmente, la importancia de la demanda también se muestra en el aumento de las transacciones -la superficie negociada aumentó un $50 \%$ cada año entre 1995/96 y 2005/07. En ello es menester tomar en cuenta que inquilinos o compradores extranjeros constituyen un porcentaje creciente. Mientras obtenían más o menos la tercera parte de las transacciones a principios de la década (con fluctuación anual), el capital extranjero ya acreditó el 48\% de las transacciones en el año 2007.

De esta manera, el mercado inmobiliario de México se ha vuelto destino de inversores globales. Un estudio de la "Sociedad Alemana de Fondos Inmobiliarios" (DEGI, 2008) identifica la Ciudad de México como el mejor lugar para invertir en América Latina (rango: 62; São Paulo: rango 67), con réditos esperables de 7.5\%, lo que es por arriba del promedio de las ciudades analizadas (6.0\%), mientras el riesgo se estima solamente muy poco encima del promedio. Con eso, calibrando los riesgos, las posibilidades de ganancia de una inversión se clasifican casi iguales que aquellos en Praga, Atenas o Auckland. Como consecuencia de esta previsión, el 
mercado mexicano atrae a inversores institucionales: Según CB Richard Ellis, más o menos 1,4\% de las inversiones institucionales a nivel mundial se hacen en México -más que en Suiza y sólo un poco menos que en Corea del Sur o Hong-Kong (Alvarado Guerrero, 2006).

El círculo se cierra: "Las compañías extranjeras son un componente significativo de la demanda total. Además, las empresas internacionales son más selectivas y solicitan un ajustes específico para sus necesidades, elevando el mercado de edificios de Clase A" dijo Claudio Tanco, entonces dirigente de Corporate Real Estate, HSBC México (citado en Mackenzie Gleason, 2005, pp. 33). Igualmente, Reinhard Kutscher, portavoz de la Junta Directiva de la Hamburger Union Investment Real Estate AG (previamente DIFA Deutsche Immobilien Fonds AG, nota de prensa DIFA, mayo del 2006, traducción propia) informó que "oficinas de alta calidad, que satisfacen a las exigencias de empresas internacionales, están en el foco". En una declaración similar el fondo alemán UniImmo Global (2007, p. 7; traducción propia) declara que "más que todo (son) empresas de los sectores de servicios financieros y farmacéuticos, como también empresas extranjeras, que se establecieron nuevamente en la Ciudad de México, han estado activas". Por eso, no sorprende entonces, que para la Union Investment Real Estate AG, "México está en la lista de compras" (Die Welt, 4 de octubre del 2008, traducción propia). Desde 2005, cuando DIFA, como primer fondo inmobiliario, invirtió en América Latina y compró con 102 millones de dólares un 30\% de la Torre Mayor en el Paseo de la Reforma, la sociedad ha invertido fuertemente en México. Adquirió, por ejemplo, el rascacielos de oficinas "Reforma 265", que está ubicado al lado de la Bolsa mexicana y que tiene al Bank of America como inquilino más prominente. Según los datos del fondo, UnImmo Global posee inmuebles (o parte de ellos) en México (sobre todo en la Ciudad de México) con un valor de 206 millones de Euros (2009), lo que representa el 8\% de todas sus inversiones y hace de México, después de Japón e Inglaterra, al tercer mercado extranjero del fondo. En cuanto a los réditos, México ha sido el mercado más lucrativo, con réditos de 19\% en 2008 y 10\% en 2009 (UnImmo Global, 2009, 2010).

\section{La transnacionalización de espacios urbanos (I): actores}

Las inversiones hechas por la Union Investment Real Estate AG en México, y las ganancias que hace con ellos, son un ejemplo para lo que en la literatura científica se describe como la internacionalización de los mercados inmobiliarios. Bajo esa noción más que todo se entiende la extensión del radio de acción de actores centrales: Inversores institucionales esparcen sus inversiones sobre los mercados de varios países. Así, los inmuebles llegaron a ser "móviles”, es decir, se convirtieron en objetos de inversión, negociados a nivel mundial. Esto va acompañado por una creciente movilidad de agencias inmobiliarias grandes, que también empezaron a actuar a nivel mundial. 
No obstante cabe la duda si "internacionalización” es el concepto adecuado para comprender los cambios en la economía inmobiliaria y las nuevas dinámicas del desarrollo urbano causados por ellos. Esta duda emerge de una diferencia conceptual entre actividades internacionales y transnacionales que se ha establecido en las literaturas sobre la migración transfronteriza y sobre las empresas transnacionales. Mientras actividades internacionales se desarrollan entre Estados nacionales, por lo cual son interestatales (la empresa internacional, p.e., tiene su producción integrada nacionalmente, mientras al mismo tiempo recurre a sucursales y proveedores extranjeros), las actividades transnacionales se localizan tanto entre Estados como dentro de los mismos. Dicken (2007) sostiene, por ejemplo, que la globalización económica está caracterizada por redes de producción que son marcadas por una integración transfronteriza y funcional. Sassen (2006), a su vez, ha sugerido que la etapa corriente del capitalismo está caracterizada por una "desnacionalización", término con el cual caracteriza una transformación en cuya marcha la conexión entre autoridad y Estado nacional pierde su exclusivismo. Nuevas formas privadas de autoridad aparecen generándose y ejerciéndose en territorios limitados por actores, que ya no se pueden adjudicar a un Estado nacional. Ciudades globales son ejemplos paradigmáticos de tales espacios nuevos, en que prácticas sociales, regulaciones, instituciones $\mathrm{u}$ ordenaciones temporales de identificaciones nacionales $\mathrm{y}$ también desnacionalizadas se encuentran.

Basándome en estos conceptos, a continuación desarrollaré el argumento de que el mercado de oficinas de primera clase o, dicho de otra manera, los espacios de la ciudad global en la Ciudad de México, están en un proceso de transnacionalización. Partiendo de la noción de Smith (2001, p. 70), quien define las ciudades transnacionales como "creaciones humanas mejor entendidas como lugares de agencia multicentrada, si no descentrada", sostengo que un espacio urbano transnacional es un espacio:

a producido por actores cuyas prácticas se desarrollan a una escala transfronteriza, por lo cual carecen de una identidad nacional claramente demarcada; y

b producido según reglas y normas que tampoco se relacionan con una localización nacional particular.

Como estudio de caso se eligió a la Torre Mayor en el Paseo de la Reforma, no solamente porque este edificio destaca físicamente del paisaje urbano (con sus 230 metros, es el edificio más alto de América Latina), sino porque también es un edificio simbólico para la formación de ciudad global en la Ciudad de México. Si una ciudad global se define como un nodo desde donde se gestionan los múltiples flujos globales, la Torre Mayor forma parte de la infraestructura física indispensable para estas operaciones. La lista de los inquilinos (más adelante) demuestra que la Torre Mayor es uno de estos nodos donde confluyen los flujos transfronterizos de las empresas globales. 
La Torre Mayor ostenta 77.000 metros cuadrados de superficie de oficinas de la categoría A+. El desarrollador es Reichmann International, una empresa de Paul Reichmann, quien, por su parte, en unión con sus hermanos, ha sido dueño de Olympia and York, "the cream of the industry" hasta el colapso en los tempranos años noventa (Fainstein, 2001, p. 160). Entre otras cosas, la empresa había realizado los proyectos First Canadian Place (1974) -el edificio más alto de Toronto-, World Financial Center en Battery Park City en Nueva York (1986-1988), como también Canary Wharf en los barrios portuarios de Londres (1992). A pesar de que su empresa esté registrada en Toronto, a Reichmann se le identifica por sus actividades en varios países, o por sus éxitos en Norteamérica, o por el fracaso de Canary Wharf en Londres, o también por sus prácticas económicas innovadoras, las cuales implican préstamos fiscales muy arriesgados, Public-Private Partnerships y técnicas nuevas y eficientes de construcción (Fainstein, 2001, p. 160). Reichmann posee un 70\% de la Torre Mayor. El 30\% restante pertenece al Fonds Union Investment Real Estate AG - el cual, aunque tenga su sede en Hamburgo, Alemania, no necesariamente maneja "capital alemán”, tampoco realiza, como se ha mencionado, sus negocios principales en Alemania. El diseño de la Torre Mayor es de Zeidler Partnerships Architects, el 76 despacho de arquitectura más grande, con una renta anual de 30-39 millones de dólares (Building Design, 2008, p. 12). Zeidler Partnerships Architects tiene su sede principal también en Toronto, mientras que otros despachos de sus oficinas se ubican en Calgary, Vancouver, Victoria, Beijing, Berlín, Londres y West Palm Beach. Hasta los años noventa, la mayor parte de los edificios se realizó en los Estados Unidos y en Canadá, pero desde entonces, la empresa, según informaciones propias, ha ensanchado sus actividades a Beijing, Seúl, Moscú, Colonia, Hannover, Dubai, Tel Aviv, Londres y a la isla del Caribe Nevis.

En este contexto cabe indicar que la arquitectura de la Torre Mayor no es específica -el edificio podría estar ubicado en cualquiera de las ciudades mencionadas (mientras tanto se busca un punto de enlace al hecho local con el nombre: Torre Mayor era el templo más importante de Tenochtitlán, la capital del imperio azteca, conquistada por los españoles). Resulta que las torres modernas de oficinas, hechas de acero y cristal, no solamente son fundamentos básicos para el global business, juntos a sus imágenes, difundidas en todo el mundo, también deben expresar el discurso dominante sobre la globalización, la competencia de ciudades y la centralidad para que una cierta ciudad pueda participar exitosamente en este global business (Grubbauer, 2009). En este sentido, Paul Reichmann dijo en una rueda de prensa con el entonces Jefe de Gobierno del Distrito Federal, Manuel López Obrador, que la Torre Mayor era la representación física de la fuerza económica de México (Voice of America, 29 de agosto del 2002).

El planeamiento arquitectónico en detalle se realizó por Adamson Associates (que tienen oficinas en Toronto, Nueva York, Las Vegas, Los Ángeles y Londres), quienes, entre otras cosas, tomaron (y toman) parte en la construcción de la sede principal de Goldman-Sachs, del Hearst Tower, además de la nueva construcción del World Trade Center. En la Ciudad de México, el despacho de arquitectura Idea 
Asociados de México tenía la responsabilidad de la realización inmediata. Las obras de construcción también se realizaron por una empresa mexicana: A.D. Tec Gerencia de Construcción. Aunque la empresa tenga mucha experiencia en la creación de inmuebles modernos de oficina (entre otros, la Bolsa Mexicana y el Citibank Building) y con contratantes extranjeros, su actividad se limita a México.

Cushman \& Wakefield, una empresa de consultoría inmobiliaria de Nueva York, que se expandió globalmente a partir de los años 1990 y ahora mantiene oficinas en todo el mundo (también en la Ciudad de México), se subcontrató para el alquiler de las superficies de la oficina de la Torre Mayor. A pesar de su origen en Nueva York, hoy en día Cushman \& Wakefield ya no tiene un centro o una identidad nacional, y eso no solamente porque sus oficinas están dispersas a nivel mundial, más importante es que el conocimiento de cómo hacer negocios (obviamente el capital más importante para una empresa de consultoría) no se adquiere en una oficina principal, sino en redes relacionales, las cuales encadenan las ubicaciones a nivel global (Beaverstock, 2004; Faulconbridge y Muzio, 2007).

En cuanto a los usuarios de la Torre Mayor, uno de los primeros inquilinos fue Deloitte \& Touche, una de las empresas de contabilidad y asesoramiento de empresas "big four". Deloitte \& Touche alquilaron más que la cuarta parte de la superficie de oficinas de todo el edificio por 10 años. Deloitte $\&$ Touche es, según el autorretrato en la página web de la empresa, "(una) marca bajo la cual decenas de miles de dedicados profesionales en empresas independientes en todo el mundo colaboran. (...) las firmas que son miembros son entidades separadas y distintas legalmente". Por ende, Deloitte \& Touche no tiene ni una casa matriz para todas sus oficinas en el mundo, ni una identidad nacional. También, Deloitte \& Touche destaca como empresa transnacional porque presta servicios a muchas de las empresas globales en México. El 24 por ciento de las empresas enlistadas en la BMV (220 de las 300 más grandes de México), obtiene sus servicios de auditoría de esta empresa, mientras 66 de las 100 empresas más grandes de México obtienen servicios legales de Deloitte. Entre los clientes globales destacan, p.e., GM, Grupo Financiero BBVA-Bancomer y Grupo Financiero Santander. Deloitte \& Touche es, por ende, un ejemplo clásico para una intersección entre la "World City Network" (Taylor 2004) y varias cadenas productivas (Parnreiter 2010). Otros inquilinos con un perfil transnacional son Santander Global Property (cuyo dueño, Banco Santander, es, según los "Forbes Global 2000", la empresa novena en el mundo), Hewlett Packard (36), Barclays (77), Apple (113) y Western Union (908). En total, alrededor de cuatro quintos de los inquilinos son empresas extranjeras.

Así, tanto los productores de la Torre Mayor como sus usuarios son en su mayoría actores que ni son mexicanos ni tienen otra identidad nacional terminante. $\mathrm{Al}$ revés, corresponden con lo que caracteriza a un consorcio transnacional: "El poder para coordinar y controlar las operaciones en más de un país” (Dicken 2007, p. 106). Por ende, se cumple con el primer criterio establecido arriba para definir espacios transnacionales - la Torre Mayor es un espacio urbano producido por acto- 
res cuyas practicas se desarrollan a una escala transfronteriza, por lo cual carecen de una identidad nacional claramente demarcada. Existen, sin embargo, más razones para llamar a la Torre Mayor un edificio transnacional. Mi conceptualización de espacios transnacionales incluye un segundo criterio, que se relaciona con las reglas y normas que guiaron la producción y que guían el uso de dicho espacio urbano. Por ende, en lo que sigue se analizan los cambios en la gestión urbana y en los procesos de planificación que hicieron posible la construcción de la Torre Mayor y otros megaproyectos.

\section{La transnacionalización de espacios urbanos (II): reglas}

En la planificación y construcción de la Torre Mayor se aplicaron instrumentos y reglas de la gestión urbana, que fueron introducidos recientemente y que difieren claramente de los instrumentos y prácticas utilizadas hasta los 80 s. La gestión de los proyectos urbanos importantes desde los 80 s (como Santa Fe -ver Jones y MorenoCarranco, 2007 y Pérez Negrete, 2009-, o el llamado rescate del Centro Histórico -ver Davis, 2006-, o la actual remodelación del Paseo de la Reforma -ver Terrazas \& Ipiña, 2007), está caracterizada por mecanismos de planificación selectiva y ad hoc, con el fin de flexibilizar y simplificar los procedimientos para la autorización de proyectos inmobiliarios.

Un primer paso en esta dirección fue la introducción de los llamados ZEDECs (Zona Especial de Desarrollo Controlado) en los 80s, que fueron fundamentales en el desarrollo del nuevo CBD en Santa Fe, porque representan "un instrumento de planeación de usos del suelo ad hoc", como lo puso Jorge Gamboa de Buen (2006, p. 5), quien en este entonces fue titular de la Secretaría de Desarrollo Urbano y Vivienda (SEDUVI) y quien hoy en día es director del Grupo Danhos, uno de los desarrolladores más importantes de México. Así, los ZEDECs permitieron alejar la planeación de la visión global de los Planes Maestros para hacerla selectiva y adaptada a casos particulares. Según Garza (1999, p. 165), este cambio en la gestión urbana resultó de la convicción de que no existe una necesidad "por planes maestros que identifiquen estrategias a ser seguidas en el mediano y largo plazo; más bien, las necesidades del mercado inmobilario determinan los proyectos que se llevarán a cabo y los usos de suelo requeridos”. Así, con la introducción de los ZEDECs se ha, en las palabras de Gambo de Buen (1994, p. 130), "logrado, por primera vez en la historia de la ciudad, ajustar los precios al potencial de desarrollo".

Otro paso para flexibilizar y simplificar los procedimientos para la autorización de proyectos inmobiliarios fue la introducción del llamado "Sistema de transferencia de potencialidad de desarrollo urbano”, que permitió ceder los derechos excedentes (o totales) de intensidad de construcción que le correspondan a un propietario, en favor de un tercero si la parte receptora está considerada como un área con potencial para crecimiento económico o de integración metropolitana. Cabe subrayar que el desarrollo hacia la flexibilización de la planificación continuó con los gobiernos del PRD a partir de 1997. A pesar de que formalmente se atuvo a los 
Planes Maestros (llamados "Programa General de Desarrollo Urbano") tanto López Obrador (2000-2005) como su sucesor Ebrard Casaubón (desde 2006) fueron guiados por la idea de que una gestión urbana eficiente debería disponer de "mecanismos ágiles de gestión, (...) planeación, regulación, fomento, control, vigilancia, coordinación y concertación”, como lo afirmó Arturo Aispuro (Aispuro, 2007, p. 6), quien en los tiempos de Gamboa de Buen como titular de la SEDUVI fue Director General de Desarrollo Urbano, y quien luego asumió el cargo de vicepresidente de desarrollo en la empresa Reichmann International en 1994, para regresar en 2006 a la SEDUVI como titular.

Un aspecto clave en aumentar la selectividad y flexibilidad de la planeación urbana fue la creación de los llamados "Corredores de Inversión y Desarrollo" (CIDs) como áreas de (aspirado) crecimiento económico. El CID más importante es el Paseo de la Reforma, que ha perdido mucho de su importancia económica en los 80 s y en los 90s, para recuperar su dinamismo a partir de la construcción de la Torre Mayor. Para fomentar el desarrollo inmobiliario en el Paseo de la Reforma y en los demás CIDs, el gobierno de Casaubón instituyó formalmente las prácticas ya establecidas: estímulos fiscales para empresas desarrolladoras, una adicional simplificación de las regulaciones (p.e. el llamado "one-stop shopping" para obtener la autorización para proyectos de construcción), tanto como una flexibilización aumentada de nuevo (las decisiones en cuanto el uso del suelo o la altura de edificios deberían ser, p.e., tomadas complacientemente) y una centralización de la toma de decisiones sobre proyectos urbanos en las manos del titular de la SEDUVI, quitándole de esta manera a las delegaciones el poder de decisión (GDF, 2008).

Finalmente, en 2010 se revisó la Ley de Desarrollo Urbano del Distrito Federal, con el fin de reducir -o hasta excluir- la participación ciudadana en las decisiones de planeación del desarrollo urbano (Pradilla Cobos, 2010). Eso vale sobre todo para las "Áreas de Gestión Estratégica", que son definidas como zonas o corredores de gran importancia económica. Allá, las decisiones sobre intervenciones urbanísticas se tomarán por un comité en el cual el Gobierno del Distrito Federal tiene la mayoría de los votos. Ahora, es preciso indicar también que la nueva ley solamente formaliza las políticas ya habituales, hechas posibles entre otras cosas por la creación de los CIDs: En el caso del Paseo de la Reforma, p.e., el Plan Maestro estableció una altura máxima para los edificios de 25 pisos. Sin embargo, 10 de los 14 proyectos inmobiliarios que están planificados o en construcción rebasarán este límite.

En cuanto a la relación entre los cambios en la planeación urbana descritos aquí y la Torre Mayor hay que subrayar dos aspectos. Primero, según los medios locales el Gobierno del Distrito Federal facilitó la aprobación de los planes a través de un manejo muy flexible de las leyes y regulaciones vigentes. Con sus 55 pisos, la Torre Mayor sobrepasó, p.e. la altura máxima determinada en ese entonces en el reglamento de zonificación, que previó solamente 38 pisos. No es, por ende, por casualidad que Arturo Aispuro, en ese entonces titular de la SEDUVI, acompañó en un discurso pronunciado en frente del cuarto congreso del ADOC (Arquitectos, Directores Responsables de Obra, Corresponsables y Peritos en Desarrollo Urbano) 
la promesa de simplificar "los procedimientos para la autorización" de proyectos urbanos con una fotografía de la Torre Mayor (Aispuro, 2007).

Segundo, y más importante, gracias al éxito económico de la Torre Mayor, las políticas aplicadas en su construcción se convirtieron en un modelo para la gestión urbana futura. Esta está, a más tardar con el Gobierno de López Obrador, dominada por la estrategia de "property led development". Según el actual titular de la SEDUVI, Leal Fernández, "en el Gobierno del Distrito Federal nos hemos preparado para que (...) se haga del desarrollo inmobiliario un verdadero instrumento de reactivación económica” (citado en Galarza, 2009). Ahora, para atraer inversiones en el desarrollo inmobiliario, el Gobierno del Distrito Federal ofrece varios incentivos para las empresas desarrolladoras. En el caso de la Torre Mayor, se condonó a la empresa Reichmann International el pago del impuesto predial durante cinco años, "como una forma de incentivar la inversión privada" (Gerald Ricker, en este entonces director general de la oficina en la Ciudad de México, citado en La Jornada, 27/06/2002). Los gobiernos del PRD no se cansan de repetir que estos incentivos salen a cuenta para el Distrito Federal, porque por cada peso que el Gobierno invirtió en el Paseo de la Reforma, la iniciativa privada puso 20 (SEDUVI 2007). Según la Secretaría, en el Paseo de la Reforma siete proyectos grandes están terminados o en construcción, mientras otros siete están autorizados o en gestión (noviembre 2010). En sus esfuerzos de ganar apoyo para las estrategias de "property led development", el Gobierno del Distrito Federal utiliza la Torre Mayor como un elemento discursivo importante. El éxito económico de este edificio (todas sus oficinas están rentadas y la mayoría de los inquilinos son de empresas globales que gozan de mucho prestigio en México) lo transformó en un modelo para todo el $\mathrm{Pa}$ seo de la Reforma y otros CIDs. Para el entonces jefe de gobierno, López Obrador, la Torre Mayor muestra que se puede invertir en el Distrito Federal, porque no falta el apoyo político (La Jornada, 27/06/2002), mientras SEDUVI indica que "(u) n ejemplo exitoso en nuestra capital es el caso del Paseo de la Reforma. Tras haber sufrido el abandono de muchos de sus inmuebles después de los sismos de 1985 esta hermosa avenida fue recuperada al reforzar su infraestructura y otorgar estímulos fiscales a los desarrolladores para fomentar las inversiones" (SEDUVI 2007). La Torre Mayor es, en breve, pintada como "(l)a punta de lanza de esta cascada de inversiones en la histórica avenida” (El Universal, 22/07/2008).

En el contexto del debate sobre la transnacionalización de espacios urbanos es importante subrayar que los mecanismos de la planeación urbana flexible, selectiva y ad hoc, que en el Distrito Federal se han introducido poco a poco a partir de la segunda mitad de los 80 s, son elementos de una estrategia que internacionalmente se conoce como "Planeación Estratégica". Con este término se nombra a un nuevo estándar global de planeación, que, según UN Habitat (2009, xxiv), enfoca solamente "aspectos o áreas que son estratégicas o importantes para los objetivos del plan general". En su totalidad, son megaproyectos como la provisión de infraestructura (transportación, comunicación, energía), la regeneración de antiguas áreas industriales, la creación de nuevos CBDs, o complejos de uso mixto (Burgess y Car- 
mona, 2009). Lo importante aquí es que la implementación de los mecanismos de la Planeación Estratégica ha sido "universal” (Burgess y Carmona, 2009, p. 12) -a escala mundial "(una) nueva forma de planificación de ciudades, a través de grandes intervenciones urbanas ha emergido, la cual puede ser mejor definida como "planificación a través de grandes proyectos"” (Carmona, 2009, p. 7).

Sin embargo, la Planeación Estratégica es universal no sólo porque se impuso en muchas ciudades a nivel global. Más aún, la Planeación Estratégica se está desarrollando en las redes transfronterizas de las Naciones Unidas y el Banco Mundial. El concepto nació con el "Urban Management Programme" del Banco Mundial y el "United Nations Centre for Human Settlements" (UN-Habitat hoy en día), cuyo objetivo fue, entre otras cosas, "promover el pensamiento estratégico en planificación” (UN Habitat, 2009, p. 66). Otra fuente de la planificación estratégica son las llamadas "City Development Strategies" (CDS) que son desarrolladas a través de "The Cities Alliance", fundada en 1999 por UN Habitat y el Banco Mundial. Por consecuencia, "(el) préstamo internacional de estas ideas" (UN Habitat, 2009, p. 15) es una práctica común, por lo cual la planificación estratégica se ha difundido a nivel mundial.

Otro aspecto que permite tomar a la Torre Mayor como un espacio urbano transnacionalizado es la tramitación de los alquileres en dólares. Se sabe bien que el dinero no solamente sirve como medio de cambio, necesario para el funcionamiento de mercados, sino que también impone -a través de las políticas monetarias- normas muy importantes para el cómo del funcionamiento de los mercados. Un ejemplo es la fijación del tipo de cambio. Inversionistas en México, p.e., siempre corren el riesgo de una devaluación -riesgo que tratan de hurtar mediante la dolarización de una parte específica del mercado inmobiliario: si el alquiler para oficinas de primera clase (como en la Torre Mayor) se paga en dólares, los propietarios e inversores pueden reducir el riesgo de desvaloración. Dicho de otra manera: La dolarización hace posible, entonces, que los inversionistas ganen la recompensa de inversiones arriesgadas en forma de réditos más altos (el rédito en la Ciudad de México es de 7.5\%, más o menos el doble que en Nueva York-DEGI 2008, p. 43), mientras, al mismo tiempo, neutralizan una parte del riesgo de la inversión. Esta es una práctica muy común: Lorena Fuertes, Executive Vice President de Jones Lang Lasalle México, calcula que alrededor del $80 \%$ de los contratos sobre inmuebles de oficinas, inmuebles residenciales e inmuebles comerciales se sella en dólares. Para los dueños de la Torre Mayor, la dolarización salió a cuenta: sin ella, hubieran tenido un déficit de ingresos de casi 20 millones de dólares por causa de la devaluación del Peso mexicano frente al dólar, lo que equivale al 6\% de las inversiones.

Así, los dos ejemplos tratados aquí -los mecanismos de planificación y la tramitación de los alquileres en dólares- indican que en cuanto a las "reglas del juego" en el mercado inmobiliario también se observa una desnacionalización de las normas y estándares que regulan la producción de los espacios urbanos globales. La infraestructura física de la ciudad global, es decir, edificios como la Torre Mayor o similares inmuebles en el Paseo de la Reforma o en Santa Fe, está producida 
según reglas que cada vez menos se relacionan con una localización nacional particular. Lógicamente, los actores extranjeros en Ciudad de México se someten a leyes mexicanas - pero eso ni con mucho comprueba que el mercado es "nacional". Precisamente, el derecho inmobiliario en México ejemplifica lo que Sassen (2006) llama la "desnacionalización de la fijación de normas", y lo que Jones Lang Lasalle comprende como un impulso para los gobiernos de "modernizar" prácticas burocráticas. Según esta empresa inmobiliaria, la globalización "creó un incentivo a los gobiernos a racionalizar las práctivas burocráticas que impiden a los extranjeros inyectar capital” (2008, p. 2). La Constitución mexicana posrevolucionaria del 1917 contenía reglas que privaron a extranjeros de comprar suelo o inmuebles en México, lo que obviamente es un obstáculo para la inversión extranjera. Al negociar el TLC, el Gobierno de México obedientemente "modernizó” el artículo 27 de la Constitución para "adaptarse" a estándares internacionales y para facilitar el boom inmobiliario que comenzó con la construcción de Santa Fe. Así, los desarrolladores de la Torre Mayor se sometieron a leyes mexicanas - pero estas leyes hoy en día son cada vez menos "mexicanas".

\section{Conclusiones}

Mediante un análisis detallado de la estructura espacial de sedes de empresas en la Ciudad de México, se demostró que la transformación de mercados inmobiliarios urbanos se motiva esencialmente por la "fuerza motriz" de procesos de la formación de la ciudad global. Por un lado, la presencia cada vez más grande de empresas globales (principalmente, pero no sólo, en el sector de los servicios al productor) aumenta la demanda de inmuebles de oficinas de alta calidad, que no existían en el volumen demandado en las ciudades de la semiperiferia de la economía global. Por otro lado, liberalizaciones amplias de mercados financieros y del suelo, en conjunto con una crisis de inversiones productivas, desembocaron en que más y más capital busque posibilidades de inversión en mercados inmobiliarios florecientes en todo el mundo.

Referente a la Ciudad de México, el resultado es un boom de construcción, financiado más que todo por inversiones extranjeras, principalmente en el sector de inmuebles de oficinas de máxima calidad. En forma de la constitución de una nueva geografía corporativa, se realiza una transformación y remodelación de la estructura arquitectónica de la ciudad. Un ejemplo clásico es la construcción del nuevo CBD en Santa Fe, y otro es la actual remodelación y recuperación del Paseo de la Reforma, que comenzó con la construcción de la Torre Mayor.

En la segunda parte del texto se argumentó que las transformaciones de los mercados inmobiliarios y de los espacios urbanos analizados aquí se comprenden mejor como una transnacionalización en vez de “internacionalización”. La Torre Mayor es un edificio que fue producido y está utilizado mayoritariamente por actores sin una identidad nacional claramente demarcada, mientras las discusión de los nuevos mecanismos de planificación en el Distrito Federal y el ejemplo de la dolarización de partes del mercado inmobiliario mostraron que tanto la producción y el uso de 
la torre están - por lo menos parcialmente- regulados por normas desnacionalizadas. La Torre Mayor es, por ende, no solamente un espacio de la ciudad global, sino también un espacio transnacionalizado que "aunque se inserta en el espacio físico del territorio nacional, puede tener poco que ver con el contexto circundante" (Sassen, 2008, p. 394). O, en las palabras de Smith (2001, p. 70), es parte de la red de ciudades transnacionalizadas, que se comprende como "creaciones humanas mejor entendidas como lugares de agencia multicentrada, si no descentrada”. IEURE

\section{Referencias bibliográficas}

Coronel, A. \& Arturo, J. (2007). Nuevo Orden Urbano en el Distrito Federal. Ponencia presentada en el IV Congreso ADOC, AC, septiembre, Distrito Federal, México.

Alvarado Guerrero, A. (2006). El Mercado de Oficinas en la Ciudad de México. Evolución y Perspectivas. Extraído el 02 de diciembre, 2008, de http://diplomadociudaddemexico.com/blog/wpcontent/uploads/modulo2/OficinasMexico2006CBREAgustinAlvarado.pdf

Beaverstock, J. V. (2004). Managing across borders: knowledge management and expatriation in professional legal service firms. Journal of Economic Geography, No. 4, pp. 157-179.

Bernardes Da Silva, A. \& Castillo, R. (2010). Dinámicas metropolitanas en la era de la globalización: la promoción inmobiliaria para empresas en la ciudad de São Paulo, Brasil. Eure, Vol. 33, No. 98, pp. 45-56.

Building Design (2008). 2008 World Architecture 100. London.

Burgess, R. \& Carmona, M. (2009). The shift of master planning to strategic planning. En M. Carmona (Ed.). Planning through Projects: Moving from Master Planning to Strategic Planning. 30 Cities. Amsterdam: Techne Press.

Carmona, M. (2009). Introduction. En M. Carmona (Ed.). Planning through Projects: Moving from Master Planning to Strategic Planning. 30 Cities. Amsterdam: Techne Press.

Colliers International (varios años). Real Estate Market Overview. Mexico City.

Colliers International (varios años). The Knowledge Report.

Cushman \& Wakefield (varios años). Marketbeat series Mexico City.

DEGI (Deutsche Gesellschaft für Immobilienfonds) (2008). Global Outlook. Immobilieninvestments 2008/2009. Frankfurt: Main.

Davis, D. (2006). Conflict, Cooperation, and Convergence: Globalization and the Politics of Downtown Development in Mexico City. Research in Political Sociology, No. 15, pp. 143-178.

Dicken, P. (2007). Global Shift: Mapping the Changing Contours of the World Economy. London: The Guilford Press.

Expansión (varios años). Las 500 empresas más importantes de México. Mexico City.

Fainstein, S.S. (2001). The City Builders: Property Development in New York and London, 1980-2000. Kansas: Lawrence.

Faulconbridge, J.R. \& Muzio, D. (2007). Reinserting the professional into the study of professional service firms. Global Networks, Vol. 7, No. 3, pp. 249-270.

Fuchs, M. \& Scharmanski, A. (2009). Counteracting path dependencies: 'Rational' investment decisions in the globalizing commercial property market. Environment and Planning A, Vol. 41, No. 11, pp. 2724-2740.

Gobierno del Distrito Federal (GDF) (2008). Gaceta official no. 248. 
Galarza, S. (2009). Abren inversiones en Paseo de la Reforma por 9,000 mdp. Extraído el 02 junio, 2010, de http://hogaresnoticia.com/index.php?option=com_content\&view=article\&id=454:i nmobiliario\&catid $=22$ : inmobiliario\&Itemid $=47$

Gamboa de Buen, J. (1994). Ciudad de México, Una Visión. México: Fondo De Cultura Económica.

Gamboa de Buen, J. (2006). The Santa Fe node: Its beginnings. Urban Age Mexico City Conference. Extraido el 09 Febrero, 2009 de www.urban-age.net

Garza, G. (1999). Global economy, metropolitan dynamics and urban policies in Mexico. Cities, Vol. 16, No. 3, pp. 149-170.

Grant, R. \& Nijman, J. (2002). Globalization and the Corporate Geography of Cities in the Less-Developed World. Annals of the Association of American Geographers, Vol. 92, No. 2, pp. 320-340.

Grubbauer, M. (2009). The high-rise office tower as a global type: exploring the architectural world of Getty Images and Co. En Guggenheim, M. y Söderström, O. (Eds.). Reshaping Cities: How Global Mobility Transforms Architecture and Urban Form. London: Routledge.

Haila, A. (2000). Real Estate in Global Cities: Singapore and Hong Kong as Property States. Urban Studies, Vol. 37, No. 12, pp. 2241-2256.

Han, S.S. (2005). Global city making in Singapore: a real estate perspective. Progress in Planning, Vol. 64, No. 2, pp. 69-175.

Harvey, D. (1985). The Urbanization of Capital. Oxford: Blackwell.

INEGI (Instituto Nacional de Estadísticas, Geografía e Informática) (2004). Censos Económicos 2004. Aguascalientes.

Jones, G. A. \& Moreno-Carranco, M. (2007). Megaprojects: beneath the pavement, excess. City: analysis of urban trends, culture, theory, policy, action, Vol. 11, No. 2, pp. 144-164.

JLL (Jones Lang Lasalle) (varios años). Mexico City. Market Dynamics. Mexico City.

JLL (Jones Lang Lasalle) (2008). From Opacity to Transparency. The Diverse World of Commercial Real Estate. Chicago.

Libertun de Duren, N. (2006). Planning à la Carte: The Location Patterns of Gated Communities around Buenos Aires in a Decentralized Planning Context. International Journal of Urban and Regional Research, Vol. 30, No. 2, pp. 308-327.

Lizieri, C.; Baum, A. \& Scott, P. (2000). Ownership, Occupation and Risk: A View of the City of London Office Market. Urban Studies, Vol. 37, No. 7, pp. 1109-1129.

Mackenzie Gleason, M. (2005). Mexico City gets premium office space: new developments like Esmeralda III and Torre Libertad show increased investment in intelligent space. Business Mexico, September, pp. 32-35.

Parnreiter, C. (2002). La Ciudad de México: el camino hacia una ciudad global. Eure, No. 85, pp. 89-119.

Parnreiter, C. (2010). Global cities in Global Commodity Chains: exploring the role of Mexico City in the geography of global economic governance. Global Networks, Vol. 10, No. 1, pp. 35-53.

Pérez Negrete, M. (2009). Santa Fe: a global enclave in Mexico City. Journal of Place Management and Development, Vol. 2, No. 1, pp. 33-40.

Pradilla Cobos, E. (2010). Participación ciudadana en el desarrollo urbano. Extraído el 20 mayo, 2010, de http://ciudadanosenred.com.mx/articulos/experto-cuestiona-ley-desarrollo-urbanoen-foro-aldf

Sabatini, F. \& Cáceres, G. (2005). Relación entre Promoción Inmobiliaria y Segregación Residencial: Giros Insospechados de la ciudad latinoamericana. Lincoln Institute of Land Policy. Documento de trabajo. 
Sassen, S. (2001). The Global City: New York. London. Tokio. Princeton: Princeton University Press.

Sassen, S. (2006). Territory, Authority, Rights. From Medieval to Global Assemblages. Princeton: Princeton University Press.

SEDUVI (2007). Una ciudad competitiva, nuevas oportunidades de inversión. Inmobiliare, No. 41, pp. 108-110.

Smith, M.P. (2001). Transnational Urbanism. Locating Globalization. Malden: Blackwell.

Taylor, P. (2004). World City Network. A global urban analysis. London: Routledge.

Terrazas, O. \& Ipiña, O. (2007). Dinámica Inmobiliaria en el cruce de dos caminos: el Paseo de la Reforma e Insurgentes en la Ciudad de México. Anuario de Espacios Urbanos, UAM/ México, pp. 39-60.

Unimmo Global (varios años). Informe anual. Extraído el 03 diciembre, 2008, de http://realestate. union-investment.de

United Nations Human Settlements Programme (UN-Habitat) (2009). Planning Sustainable Cities. Global Report on Human Settlements 2009. London: Earthscan.

Valenzuela, A. (2007). Santa Fe (México): Megaproyectos para una ciudad dividida. Cuadernos Geográficos, Vol. 40, No. 1, pp. 53-66.

Yusuf, S. \& Wu, W. (2002). Pathways to a World City: Shanghai Rising in an Era of Globalisation. Urban Studies, Vol. 39, No. 7, pp. 1213-1240. 